\title{
Carnets
}

Revue électronique d'études françaises de l'APEF

Deuxième série - 9 | 2017

Reconnaissances et légitimité en français

\section{La légitimité des littératures francophones}

Aspects théoriques, critiques et didactiques

José Domingues de Almeida

\section{OpenEdition}

Journals

Édition électronique

URL : http://journals.openedition.org/carnets/2123

DOI : $10.4000 /$ carnets. 2123

ISSN : 1646-7698

Éditeur

APEF

Référence électronique

José Domingues de Almeida, "La légitimité des littératures francophones », Carnets [En ligne], Deuxième série - 9 | 2017, mis en ligne le 31 janvier 2017, consulté le 01 mai 2019. URL : http:// journals.openedition.org/carnets/2123; DOI : 10.4000/carnets.2123

Ce document a été généré automatiquement le 1 mai 2019.

\section{(c) (i) \&}

Carnets est mis à disposition selon les termes de la licence Creative Commons - Atribution - Pas d'utilisation commerciale 4.0 International. 


\title{
La légitimité des littératures francophones
}

\author{
Aspects théoriques, critiques et didactiques
}

José Domingues de Almeida

1 La question de la légitimation des littératures de langue française produites en dehors $\mathrm{du}$ contexte hexagonal s'avère problématique à plus d'un titre. Pour mieux cerner les contours de notre sujet, il nous faut considérer les deux volets qu'il comporte. D'une part, les écueils historiques et identitaires auxquels ces productions littéraires sont confrontées, ainsi que les stratégies narratives et éditoriales mises en place par les littératures francophones, ou utilisées à leur endroit pour s'assurer, ou leur nier, l'accès aux instances légitimantes. D'autre part, les atouts de l'enseignement de ces textes dans leurs contextes autochtones d'abord, mais surtout dans le cadre de l'enseignementapprentissage du Français Langue Étrangère (FLE).

2 À la faveur de la notion de « légitimité » institutionnelle littéraire, de ses rites et de ses instances, nous entendons interroger la caractérisation des littératures francophones en tant que fictions à processus de légitimation distinct de celui de la littérature produite dans l'Hexagone, parce que marquées par la complexité identitaire et historique.

3 En fait, il s'agit d'abord de dégager et de décrire les conditions et les parcours de légitimation que la machine institutionnelle littéraire fait subir aux auteurs et aux ouvrages francophones afin de poser, dans un deuxième temps, les enjeux didactiques de l'introduction de ces textes dans l'enseignement-apprentissage du FLE.

4 À cet égard, pour l'exemple, le statut et les déboires très particuliers des lettres belges de langue française, ressassés dans plusieurs manifestes, - datés certes, et intimement liés au mouvement de la belgitude, mais qui n'en illustrent pas moins les conditionnements subliminaux de l'écriture littéraire en français hors de l'Hexagone -, procurent un cadre suffisamment solide de compréhension historique de notre sujet, que pour être projeté et généralisé dans les autres périphéries littéraires du français. Dans tous les cas, il est avant tout question d'une revendication historique et identitaire, mais aussi d'une volonté de réappropriation de la / sa langue. 
5 Selon les cas et les idiosyncrasies locales, les aires littéraires francophones latérales, ou totalement périphériques par rapport à l'Hexagone ou à la Métropole ont connu des stratégies et des processus particuliers d'affirmation. Évoquons le mouvement manifestaire de la belgitude, aux relents politiques, à un moment de bascule par rapport au mythe unitaire belge. Moins politique et plus complexe, vu la formation confédérale de la Suisse, la suissitude a, elle aussi, combattu un mythe unitaire, l'helvétisme, tandis que, pour le Québec, du fait de la distance du centre, le caractère particulier de sa lutte revendicative en milieu anglophone hostile, et l'engagement de ses écrivains et intellectuels, le même rapport complexé n'est ni éprouvé, ni complexé. Bien au contraire, comme le rappelait Pierre Mertens, le Québec avait déjà « récupér[é] sa langue » (AAVV, $1979 / 80: 28)$.

6 Pour le reste des vastes espaces littéraires francophones, le processus de reconnaissance est à poser diachroniquement, et est ponctué par une périodisation critique et esthétique qui va de la négritude, à la multiplicité d'approches et lectures postcoloniales, pour en arriver à la littérature-monde, et aboutir (à nouveau) au projet francophone, mais en concurrence désormais avec un agenda nettement politique de l'Organisation Internationale de la Francophonie, comme l'a bien décrit François Provenzano (2011). En fait, comme l'affirme ce théoricien, «(...) l'inscription d'une 'littérature francophone' dans l'histoire littéraire est essentiellement une affaire de représentation, chevillée aux évolutions du projet d'une 'francophonie' politique et, en particulier, aux intérêts français portés par ce projet » (idem : 37), ce qui n'en configure pas moins le dégagement d'une compartimentation problématique :

En effet, dès lors que l'on reconnaît l'existence d'un «fait littéraire francophone » distinct, sous plusieurs aspects, du "fait littéraire français ", se pose la question de l'articulation entre la prise en compte globale de ce «fait littéraire francophone " et le découpage "par zones ", qui a construit des cadres de référence suffisamment stables pour au moins trois des grandes portions de la «francophonie littéraire »: la Belgique francophone, la Suisse romande, le Québec [entendons, pas aussi stables pour le reste] (idem : 43).

7 La doxa francophone en tant qu'« ensemble des discours qui tissent un lien entre ces deux grands ensembles » (idem : 55) aurait historiquement produit des topoï à même d'assurer, voire de fonder, une rhétorique francophone qui a la vie dure si l'on considère leur résilience dans les Études Francophones, même si d'aucuns les ont nuancés et mis en contexte historique. Citons les dichotomies centre-périphéries, littérature légitime et paralittérature, langue française et surconscience linguistique, etc.

8 C'est justement dans cet éventail topique que l'on retrouvera les stratégies de légitimation, lesquelles actent la préséance institutionnelle de la France sur la production des produits symboliques en langue française. Pour des raisons qui tiennent à son poids démographique au sein de l'ensemble francophone, la France demeure un puissant " surmoi ", éditorial notamment, et une instance sourcilleuse de légitimité symbolique et littéraire qui intimide aussi bien les aires latérales que les ex-colonies, pour ne pas dire les écritures littéraires allophones, où le français fait l'objet d'une élection.

9 Manque à la Francophonie le polycentrisme éditorial et culturel dont jouissent les autres ensembles europhones. Et, de fait, Paris demeure, pour une large part, l'unique véritable centre éditorial francophone concentrant les instances, les prix, les rituels, les moyens et le prestige associés à la machine littéraire, les instances de légitimité et de reconnaissance en somme. Et c'est toujours Paris pour une bonne part qui dispense, à son 
gré, la légitimité et la reconnaissance auxquelles toutes les productions culturelles francophones aspirent, de sorte que les écrivains y sont malgré eux "réverbérés " (AAVV, 1979/80: 20). Comme le relève François Provenzano: «(...) si elles ont un potentiel littéraire, les périphéries ne peuvent l'actualiser que dans le cadre de l'influence française » $(2011: 85)$.

C'est d'ailleurs, rappelons-le, l'argument de l'attribution plus généreuse de prix littéraires français et internationaux à des écrivains non-hexagonaux qui servira de fondement, voire de pièce à conviction, dans le discours des signataires du Manifeste pour une littérature-monde en français (2007), foncièrement anti-francophone, mais qui n'a pas abouti au résultat institutionnel ou esthétique escompté. Et c'est toujours à partir du repère presque exclusivement français que Christiane Albert inscrivait naguère la scénographie discursive propre aux écrivains migrants de langue française, tout comme la traçabilité de leur reconnaissance, comme par exemple celle des littératures spécifiquement migrantes: "Ces littératures sont désormais enseignées dans de nombreuses universités tant françaises qu'étrangères, elles font l'objet de colloques, possèdent leurs revues spécialisées et sont éditées chez les plus grands éditeurs [français, s'entend] » (Albert, 2005 : 174).

11 En fait, même dans le cas des littératures « hybrides » (Sebkhi, 1999) de l'immigration en français, à mi-chemin taxinomique et identitaire entre les littératures francophones, la littérature française, voire les littératures nationales des pays d'origine, tout est question de stratégie de légitimation:

Aussi pour acquérir de la légitimité littéraire à laquelle ils aspirent et du fait de leur situation périphérique à la fois vis-à-vis de leur culture d'accueil (la culture française) [France, quand tu nous tiens !] et de celle de leur pays d'origine, les écrivains de l'immigration se trouvent donc dans la nécessité de se démarquer culturellement de ces champs constitués. Ils le font en remettant en question la notion de nationalisme littéraire et en revendiquant une identité métisse qui leur permette d'échapper à toute tentative d'étiquetage tout en expérimentant des procédés d'écritures marquées à la fois par l'hybridation et l'hétérogénéité (Albert, $2005: 176)$.

Habiba Sebkhi n'hésite pas à recourir à la métaphore juridique de l'enfant naturel vs légitime pour caractériser la difficulté qu'il y a à classer cette littérature d'un autre type, souvent en deuxième, voire troisième génération (Sebkhi, 1999). Selon elle, «L'illégitimité se situe à deux niveaux. Elle est à repérer autour et à l'intérieur de la littérature beur. Elle se situe d'abord au sein de l'institution, de manière externe ; elle se manifeste ensuite de manière interne dans le tissu narratif » (idem).

Et Christiane Albert de renchérir en pointant des collections parisiennes: "grands éditeurs français [qui] ouvrent des collections » (Albert, 2005 : 177) spécialisées dans les littératures migrantes, mais aussi francophones (Présence africaine, L'Harmattan ou Le serpent à plumes), lesquelles « accueillent les écrivains de l'immigration (...)» (idem : 178). Plus loin, Albert refrène son optimisme théorique en rappelant que «(...) les écrivains issus de l'immigration africaine sont surtout publiés chez des éditeurs ou dans des collections spécialisées dans la publication de littérature africaine; les prix littéraires qu'ils reçoivent sont surtout destinés à récompenser des œuvres francophones : Prix des Tropiques, Grand Prix littéraire d'Afrique noire, Prix du meilleur auteur africain, etc. » ( idem : 183).

14 D'autant plus que les espaces francophones ressentent inconsciemment l'absence d'un solide contrepoids à l'Hexagone, la France n'ayant pas historiquement accouché d'un 
quelconque Mexique, Brésil ou États-Unis. Ces espaces ont ainsi été souvent maintenus en état de minorisation et d'allégeance culturelle à tel point que des générations d'écrivains francophones n'ont pas éprouvé le besoin de parler de leurs réalités idiosyncrasiques, ou à partir d'elles.

Dès lors, la critique parisienne n'a bien souvent pas hésité (et n'hésite toujours pas) à octroyer de la légitimité littéraire aux textes francophones selon des figures condescendantes, telles que le trajet, la découverte et la différence. Jean-Marie Klinkenberg illustre ces trois figures par le biais des propos de critiques français recueillis dans la presse littéraire parisienne, concernant des auteurs francophones (1985). La figure du «trajet » insiste sur l'origine distante et périphérique de l'écrivain, compensée par la perspective de reconnaissance. Ainsi, de l'écrivain belge Conrad Detrez, bien avant sa naturalisation française, il a été dit en 1979 qu'il « a poussé dans l'étroit milieu d'un petit village belge » (Figaro). La « découverte » traduit le fait que la critique « découvre » un talent littéraire qui, autrement, aurait pu ne pas être mis à profit. Et l'on pourrait citer le cas de l'écrivain belge Eugène Savitzkaya qui, en 1979, sort « un livre éblouissant et doux (...) qu'il serait navrant de laisser enterrer sous je ne sais quel silence vaguement complice " (Figaro) ; voire cet autre exemple de découvreur sur le même auteur, dans Le Monde cette fois : « On commence à connaître ici Eugène Savitzkaya, qui vit en Belgique (... )». Finalement, par "différence», on reconnait l'expression d'une thématique ou de tournures stylistiques spécifiques et / ou exotiques. Il suffit d'évoquer la détection d'africanismes, de belgicismes ou d'autres topolectismes esthétiques.

Dans cette logique, on pourrait avec Anne-Rosine Delbart et Sophie Croiset affirmer que a priori et pendant longtemps - «l'étiquette de 'littérature francophone' a [eu] mauvaise presse auprès du public de langue française qui ignore[ait] les 'écrivains francophones' ou ne leur témoigne[ait] pas beaucoup de crédit littéraire jusqu'à ce qu'une consécration officielle - sur la place parisienne dans le meilleur des cas - ne les sorte du lot » (Delbart \& Croiset, $2011: 1$ ).

De son côté, François Provenzano parcourt de façon critique et décomplexée les étapes et les figures d'une doxa et d'un savoir théorique francophones dans lesquels la question stylistique s'est souvent avérée axiale et différenciatrice dans les rapports des périphéries au centre. C'est dans ce contexte qu'il faut placer la notion de "surconscience linguistique » mise en orbite par Lise Gauvin (1997), mais que Christiane Albert réfère paradoxalement aux «premiers écrivains francophones dont le français n'était pas la langue maternelle» (Albert, 2005 : 169). Pour François Provenzano, il s'agirait « (...) de s'appuyer sur un état des lieux sociolinguistiques des situations d'énonciation des 'écrivains francophones' pour faire apparaitre les réponses stylistiques originales qu'ils ont pu y apporter, les stratégies compensatoires qu'ils ont déployées face à des situations problématiques » (Provenzano, $2011: 45)$, soit l'existence d'une «'poétique générale de la francophonie, préalable à une stylistique des œuvres francophones" (ibidem), que l'on pourrait d'ailleurs apparier à une thématique spécifique, elle aussi antérieure.

Dans cette logique, puisque c'est la scène parisienne qui anime et légitime le champ littéraire en langue française, ne restent aux écrivains francophones que deux stratégies, et ce pour " ruser avec l'illégitimité », c'est-à-dire pour « s'attribuer de la légitimité », qui se traduisent soit par « une autonomisation du champ littéraire distinct par rapport à l'instance parisienne, mais qui annule la hiérarchie, donc la légitimité », soit par un « effort d'assimilation au champ parisien » (Klinkenberg, 1989). 
19 En outre, et deuxième volet de notre propos, il s'agit de considérer l'intérêt didactique de la lecture de ces littératures dans des contextes exolingues de l'enseignementapprentissage du FLE. Comme le rappelle Jean-Marc Defays et al., l'approche communicative et la perspective interculturelle ont assez rapidement remis à l'honneur l'apport de la littérature à l'enseignement-apprentissage des langues étrangères et secondes en général, et du FLE en particulier (2014: 23). Selon lui, la didactique du FLE réapprécie la pratique de la lecture littéraire dans la mesure où elle lui redécouvre simultanément des occasions d'ouverture sur le monde et sur la diversité / complexité communicatives (Cuq \& Gruca, 2002 : 384), en phase avec les directives du Cadre Commun de Référence pour les Langues (2001), et cautionne le rôle d'un genre comme le roman en tant qu'« outil efficace pour rendre l'apprenant sensible à la différence de registres formels ou familiers » (Defays et al. $2014: 25$ ).

Dès lors se pose la question de l'opportunité de l'ouverture de la didactique du FLE sur le roman francophone ou allophone. Reine Berthelot en dégage une problématique du statut du roman francophone dans le contexte de l'enseignement-apprentissage du FLE qui passe par la reconnaissance et la prise en charge didactique de sa spécificité esthétique, à savoir la relative opacité discursive et la plus grande présence de l'oralité, mais aussi l'ouverture sur un référent à thématiques distantes et distinctes de l'idiosyncrasie européenne; ce qui confère aux littératures francophones ou allophones en français un degré d'extranéité qui peut s'avérer un puissant inducteur interculturel : « cet ailleurs qui s'affirme ainsi à l'intérieur de la langue, ouvre des horizons nouveaux, tant dans la reconnaissance d'autres écritures imprégnées par leur culture d'origine que de thématiques nouvelles » (Berthelot, $2011: 43$ ).

21 Ces écrivains et ces écritures "venus d'ailleurs", - selon l'heureuse expression d'AnneRosine Delbart (2005) -, placent la langue française, et son enseignement-apprentissage en tant que langue étrangère, dans une perspective mondiale et mondialisée, à même de se départir d'une Histoire ou identité uniques ou univoques. Or, le roman francophone ou allophone en français s'avère un puissant médiateur de cultures, un outil efficace dans la déclinaison des diversités et convergences francophones, notamment par rapport à l'Hexagone et à l'Europe. Defays et al. rendent cette capacité de médiation de la sorte: "L'expérience littéraire du bilinguisme serait exportable dans la classe de FLE à la fois comme auxiliaire de l'accès à la littérature dans la langue étrangère et comme révélateur pour les apprenants de leurs propres représentations et de leurs compétences de la langue-cible» (2014: 81). Il y aurait quelque part la possibilité d'identification de l'apprenant avec une certaine distance de l'écrivain francophone ou allophone par rapport au français.

Aussi bien dans cet ouvrage collectif que chez Reine Berthelot, des noms d'écrivains et des titres de roman sont avancés dans le sens d'une prise en charge didactique de leur légitimité littéraire. Parmi eux, certains sont devenus incontournables et sont largement connus du grand public: Patrick Chamoiseau, Tahar Ben Jelloun, mais aussi des romanciers plus récents et inscrits dans des taxinomies francophones plus complexes ou hybrides: littérature migrante, beure, etc. Dès lors, d'autres noms s'affirment comme propositions de lecture, souvent détenteurs de prix littéraires décernés comme autant de signes de légitimité et de reconnaissance, tels qu'Azouz Begag, Ahmadou Kourouma ou Leïla Houari, dont les thématiques et les horizons diégétiques offrent des pistes de lecture très pertinentes, même si, de préférence, à un niveau plus avancé des descripteurs de 
l'enseignement-apprentissage du FLE (B2). On pourrait également citer des noms tels que Mina Oualdlhadj, Malika Madi, Kenan Görgün, Altay Manço, voire Fouad Laroui.

L'essai collectif La Littérature dans l'enseignement du français langue étrangère évoque l'avantage de travailler ces thématiques au goût du jour et en phase avec l'émergence des littératures-monde (AAVV, 2015 : 153) à la faveur de l'enseignement-apprentissage du FLE. En effet, «l'apprenant [y] découvre des textes mettant en jeu le déracinement, la migration, ou le devenir autre " (idem: 156). On le voit immédiatement: si les auteurs francophones ont souvent joué le jeu des stratégies stylistiques en vue de s'assurer reconnaissance et légitimité, la didactique FLE recourt actuellement à ces auteurs afin d'y faire lire des thématiques distinctes et davantage propices à une ouverture sur le monde. En outre, la présence du roman francophone et allophone en français dans l'enseignement-apprentissage du FLE implique une pratique didactique concrète et des propositions méthodologiques en vue de sa plus large mise à profit pédagogique. À cet égard, plusieurs suggestions et pratiques très pragmatiques et ciblées, ou pistes de travail en classe pour enseignants de FLE à certains stades (plutôt avancés) du Cadre Commun de Référence pour les Langues ont récemment vu le jour. Relevons l'essai de Reine Berthelot sur les Littératures francophones en classe de FLE. Pourquoi et comment les enseigner (2011) qui, dans son volet " comment ", expose plusieurs fiches thématiques de travail à partir de la lecture d'auteurs allophones, échelonnée sur plusieurs séquences et séances comportant une présentation biobibliographique, une suggestion d'extraits, de thèmes à développer et une observation de post-lecture : le quotidien chez Chadortt Djavann et l'erreur chez Agota Kristof ou Anna Moï (idem : 55-81).

Plus récemment, La Littérature dans l'Enseignement du FLE, pointe plusieurs activités didactiques pratiques pour l'apprenant: la lecture à voix haute, des mises en relation thématiques et référentielles, l'exploitation syntaxique et sémantique, l'incitation à l'écriture et à l'expression orale (AAVV, 2015 : 145-151).

Il ressort de ce qui vient d'être exposé que le littéraire (notamment le roman francophone) est loin d'avoir dit son dernier mot en didactique du FLE. Il s'avère même un outil imprévu en vue de la systématisation et de la pratique des compétences inscrites au programme et dans les descripteurs du Cadre, d'autant plus qu'il s'ouvre sur le monde et sur le dialogue interculturel, et qu'il concourt à suppléer un apprentissage en FLE uniquement fondé sur les manuels et les grammaires, où prime l'écrit artificiellement axé sur le monologue et la phrase verbale simple, et ce, par un apport, fût-il excessif, d'expressivité et d'emphase (Vigner, 2004: 57). À explorer davantage, et plus tôt, la pratique de la lecture du roman permet aussi de dépasser le simple souci communicatif pour introduire l'apprenant dans les associations symboliques et culturelles.

Force est toutefois de remarquer que les didacticiens ayant investi le domaine littéraire francophone le réduisent trop souvent à l'écriture allophone en français, laquelle ne présente pas le même degré de problématicité ou de représentativité, voire le même intérêt d'ouverture interculturelle. Il s'agirait dès lors de procurer des réalités et des univers culturels plus distants du nôtre (Afrique, Moyen-Orient, Asie), mais aussi de donner à lire des contextes d'écriture nouveaux: migration, questionnements identitaires, entre-deux ou perspectives cosmopolites et hybrides.

Dans La Littérature en FLE. État des lieux et nouvelles perspectives, Anne-Rosine Delbart plaide pour une mise à profit didactique d'écrivains très contemporains travaillant des questions identitaires de double appartenance culturelle ou de frontières, notamment les 
textes fictionnels portant sur le témoignage de vécus interculturels, et que nous évoquions plus haut. C'est là que les textes francophones gagnent une nouvelle « reconnaissance », et en tout cas une « utilité » didactique insoupçonnée.

\section{BIBLIOGRAPHIE}

AA.VV. (1979/80). Critique politique, n 4, « Ecrire de la Belgique », novembre-décembre-janvier.

AA.VV. (2007). Pour une littérature-monde en français. M. Le Bris et J. Rouaud (dir). Paris : Gallimard.

AA.VV. (2015). La Littérature dans l'enseignement du FLE. A. Godard (dir). Paris : Didier.

ALBERT, Christiane (2005). L'Immigration dans le roman francophone contemporain. Paris : Karthala.

BERTHELOT, Reine (2011). Littératures francophones en classe de FLE. Pourquoi et comment les enseigner.

Paris : L'Harmattan.

CONSEIL DE L'EUROPE (2001). Cadre Européen Commun de Référence pour les Langues : apprendre, enseigner, évaluer. Paris : Didier.

CUQ, Jean-Pierre / GRUCA, Isabelle (2002). Cours de didactique du FLE. Grenoble : PUG.

DEFAYS, Jean-Marc et al. (2014). La Littérature en FLE. État des lieux et nouvelles perspectives. Paris : Hachette Français Langue Étrangère.

DELBART, Anne-Rosine (2005). Les Exilés du langage : un siècle d'écrivains français venus d'ailleurs (1919-2000). Limoges : PULIM.

DELBART, Anne-Rosine / CROISET, Sophie (2011). « Présentation du dossier Marginalité, identité et diversité des 'littératures francophones'», Le Langage et l'Homme, vol. 46, fascicule 1 (juin), pp. 1-8.

GAUVIN, Lise (1997). « D'une langue, l'autre. La surconscience linguistique de l'écrivain

francophone ", L'écrivain francophone à la croisée des langues. Paris : Karthala, pp. 5-15.

KLINKENBERG, Jean-Marie, et al. (1985). Trajectoires : littératures et institutions au Québec et en Belgique francophone. Bruxelles : Labor.

KLINKENBERG, Jean-Marie (1989). « Le problème de la langue d'écriture dans la littérature francophone de Belgique de Verhaeren à Verheggen ", in A. Vigh (dir). L'identité culturelle dans les littératures de langue française. Paris : P. V. Pécs / ACCT, pp. 69-73.

PROVENZANO, François (2011). Vies et mort de la francophonie. Une politique française de la langue et de la littérature. Bruxelles : Les Impressions Nouvelles, coll. « Réflexions faites ».

SEBKHI, Habiba (1999). « Une littérature naturelle : le cas de la littérature 'beure'» in Itinéraires et contacts de cultures, $n^{\circ} 27$, pp. 16-27 (consulté le 13/09/2015 sur le site www.limag.refer.org).

Vigner, Gérard (2004). La Grammaire en FLE. Paris : Hachette Français Langue Étrangère. 


\section{RÉSUMÉS}

Cet article entend interroger les conditions et les stratégies d'accès aux instances de légitimité et de reconnaissance littéraire de la part des écrivains francophones périphériques contemporains publiant à Paris à partir de réalités distinctes. Par ailleurs, nous passerons en revue des questions soulevées par le recours de textes fictionnels francophones dans le cadre de l'enseignementapprentissage du français langue étrangère.

This paper intends to question the conditions and strategies of access to legitimacy and acknowledgement by contemporary peripheral Francophone writers who publish in Paris about their own different realities. Moreover, we will survey some questions raised by the use of Francophone fictional texts in the context of French Foreign Language teaching-learning process.

\section{INDEX}

Mots-clés : légitimité, reconnaissance, littératures francophones, Français Langue Étrangère (FLE), périphéries

Keywords : legitimacy, acknowledgement, Francophone literatures, French as a Foreign Language (FFL), peripheries

\section{AUTEUR}

\section{JOSÉ DOMINGUES DE ALMEIDA}

Un. Porto - APEF - ILC ML

jalmeida[at]letras.up.pt 